\title{
A New Approach to Regular \& Indeterminate Strings
}

\author{
Felipe A. Louza ${ }^{\mathrm{a}}$, Neerja Mhaskar ${ }^{\mathrm{b}, *}$, W. F. Smyth ${ }^{\mathrm{b}, \mathrm{c}, \mathrm{d}}$ \\ ${ }^{a}$ Faculty of Electrical Engineering, Federal University of Uberlândia, Brazil \\ ${ }^{b}$ Department of Computing and Software, McMaster University, Canada \\ ${ }^{c}$ Department of Informatics, King's College London, UK \\ ${ }^{d}$ School of Engineering $\&$ Information Technology, Murdoch University, Australia
}

\begin{abstract}
In this paper we propose a new, more appropriate definition of regular and indeterminate strings. A regular string is one that is "isomorphic" to a string whose entries all consist of a single letter, but which nevertheless may itself include entries containing multiple letters. A string that is not regular is said to be indeterminate. We begin by proposing a new model for the representation of strings, regular or indeterminate, then go on to describe a linear time algorithm to determine whether or not a string $\boldsymbol{x}=\boldsymbol{x}[1 . . n]$ is regular and, if so, to replace it by a lexicographically least (lex-least) string $\boldsymbol{y}$ whose entries are all single letters. Furthermore, we connect the regularity of a string to the transitive closure problem on a graph, which in our special case can be efficiently solved. We then introduce the idea of a feasible palindrome array MP of a string, and prove that every feasible MP corresponds to some (regular or indeterminate) string. We describe an algorithm that constructs a string $\boldsymbol{x}$ corresponding to given feasible MP, while ensuring that whenever possible $\boldsymbol{x}$ is regular and if so, then lex-least. A final section outlines new research directions suggested by this changed perspective on regular and indeterminate strings.

Keywords: indeterminate string, degenerate string, generalized string, transitive closure, palindrome, maximal palindrome array, Manacher's condition, reverse engineering, algorithm, stringology
\end{abstract}

\footnotetext{
${ }^{*}$ Corresponding author

Email addresses: louza@ufu.br (Felipe A. Louza), pophlin@mcmaster.ca (Neerja Mhaskar), smyth@mcmaster.ca (W. F. Smyth)
} 


\section{Introduction}

Under various names and guises, the idea of a string as something other than a sequence of single letters has been discussed for almost half a century. In 1974 Fischer \& Paterson 1] studied pattern-matching on strings $\boldsymbol{x}$ whose entries could be $\boldsymbol{d o n}$ 't-care letters; that is, letters matching any single letter in the alphabet $\Sigma$ on which the string is defined, hence matching every position in $\boldsymbol{x}$. (Thus the don't care letter $*$ would match every letter in a given alphabet $\Sigma=\{a, b, c\}$.) In 1987 Abrahamson [2] extended this model by considering pattern-matching on generalized strings whose entries could be non-empty subsets of $\Sigma$. (Thus entries $\{b\},\{a, b\},\{b, c\}$ would match each other due to the shared $b$.)

Both of these models have been intensively studied in this century, with interest fueled by applications, especially to computational biology, where as a byproduct of the DNA sequencing process and in other contexts, indeterminate letters such as $\{a, t\},\{c, g\}$ or $\{a, c, g, t\}$ may arise. The properties of strings with don't care letters, also called holes, have been intensively studied by Blanchet-Sadri, culminating in a 2008 book [3] on strings with holes, also called partial words, that summarized the contributions of her many previous research articles. In 2009 a periodicity lemma for partial words was formulated in [4].

Meanwhile Iliopoulos and various collaborators (see, for example, [ 5, , 6, 7]) studied pattern-matching and other problems, especially related to bioinformatics applications, on generalized strings (now renamed degenerate strings, or even elastic degenerate strings). Another sequence of papers [8, 9, 10, 11, 12] studied other pattern-matching algorithms on generalized strings (renamed indeterminate strings, with indeterminate letters such as $\{a, b\}$ or $\{b, c\}$ in some positions). In addition, other aspects of indeterminate strings have been studied, such as their border arrays [13], cover arrays [14], prefix arrays [15, 16] and suffix arrays [17, 13]. The computation of an indeterminate string from its prefix graph was recently considered in [18].

The traditional approach to defining an indeterminate string only relies on 
the scope of its letters irrespective of the matching relationship between them. Therefore, a string $\boldsymbol{x}=a\{a, c\} b\{a, d\} b b$ can be seen as an indeterminate string, when in essence it can be reduced to an "isomorphic" string $\boldsymbol{x}^{\prime}=a a b a b b$ that is regular (see Section 2 for definitions). It is evident that algorithms designed for indeterminate strings are slower and at times require more space for processing. Therefore, it is important to identify strings that are "truly" indeterminate. In this paper we redefine an indeterminate string in a context that we believe captures the idea in a more appropriate way - at once more general and more precise.

The outline of the paper is as follows: in Section 2 we present the necessary definitions, including in particular a model for the representation of strings that efficiently incorporates both regular and indeterminate. In Section 3, we apply this model to describe a linear time algorithm that determines whether or not a given string is regular and, if so, returns an isomorphic lexicographically least (lex-least) regular string, whose entries are all single letters. We go on to present an interesting connection between strings defined using our approach and the transitive closure of a relation, showing that for some special cases of a relation, its transitive closure can be computed in quadratic time. In Section 4 we introduce a "Reverse Engineering" problem [19, 20, 21, 22] for both regular and indeterminate strings (in our new formulation). We first show that every feasible palindrome array MP does indeed correspond to some string, regular or indeterminate. Then, following [23], we describe an algorithm that, given a feasible palindrome array MP, computes a lex-least regular string corresponding to MP, whenever that is possible; and, if not, returns a corresponding indeterminate string. Section 5 discusses open problems and questions arising from this new approach.

\section{Regular \& Indeterminate Strings}

A letter $\ell$ is a finite list of $s$ distinct characters $c_{1}, c_{2}, \ldots, c_{s}$, each drawn from a set $\Sigma$ of size $\sigma=|\Sigma|$ called the alphabet. In the case that $\Sigma$ is ordered, 
$\ell$ is said to be in normal form if its characters occur in the ascending order determined by $\Sigma$. The integer $s=s(\ell)$ is called the scope of $\ell$. If $s=1, \ell$ is said to be regular, otherwise indeterminate. Two letters $\ell_{1}, \ell_{2}$ are said to match, written $\ell_{1} \approx \ell_{2}$, if and only if $\ell_{1} \cap \ell_{2} \neq \emptyset$. In the case that matching $\ell_{1}$ and $\ell_{2}$ are both regular, we may write $\ell_{1}=\ell_{2}$.

For $n \geq 1$, a string $\boldsymbol{x}=\boldsymbol{x}[1 . . n]$ is a sequence $\boldsymbol{x}[1], \boldsymbol{x}[2], \ldots, \boldsymbol{x}[n]$ of letters, where $n=|\boldsymbol{x}|$ is the length of $\boldsymbol{x}$, and every $i \in\{1, \ldots, n\}$ is a position in $\boldsymbol{x}$. If every letter in $\boldsymbol{x}$ is in normal form, then $\boldsymbol{x}$ itself is said to be in normal form. A tuple $T=\left(i, j_{1}, j_{2}\right)$ of distinct positions $i, j_{1}, j_{2}$ in $\boldsymbol{x}$ such that

$$
\boldsymbol{x}\left[j_{1}\right] \approx \boldsymbol{x}[i] \approx \boldsymbol{x}\left[j_{2}\right]
$$

is said to be a triple. A triple $T$ is transitive if $\boldsymbol{x}\left[j_{1}\right] \approx \boldsymbol{x}\left[j_{2}\right]$, otherwise intransitive. If every triple $T$ in $\boldsymbol{x}$ is transitive, then we say that $\boldsymbol{x}$ is regular; otherwise, $\boldsymbol{x}$ is indeterminate. The scope of $\boldsymbol{x}$ is given by

$$
S(\boldsymbol{x})=\max _{i \in\{1, \ldots, n\}} s(\boldsymbol{x}[i]) .
$$

Two strings $\boldsymbol{x}$ and $\boldsymbol{y}$ of equal length $n$ are said to be isomorphic if and only if for every $i, j \in\{1, \ldots, n\}$,

$$
\boldsymbol{x}[i] \approx \boldsymbol{x}[j] \Longleftrightarrow \boldsymbol{y}[i] \approx \boldsymbol{y}[j]
$$

Note that isomorphic strings may superficially have quite different representations; for example, $\boldsymbol{y}=\{a, b\}\{b, c\}\{a, c\}$ is isomorphic to $\boldsymbol{x}=a a a$. Thus we see that the definition given here of regularity of a string $\boldsymbol{x}$ is more general, including many more strings, than the usual definition ( literature. However:

Lemma 1. Every regular string is isomorphic to a string of scope 1.

Proof. In [16, 18] and elsewhere it is observed that a regular string $\boldsymbol{x}[1 . . n]$ is equivalent to a graph $\mathcal{G}$ on vertices $\{1, \ldots, n\}$ which is a collection of cliques 
with the property that vertices $i$ and $j \neq i$ are in the same clique if and only if $\boldsymbol{x}[i] \approx \boldsymbol{x}[j]$. Now if, for every clique $\mathcal{C}$ of $\mathcal{G}$, we choose a unique regular letter $\ell_{\mathcal{C}}$ and, corresponding to every position $i$ in $\mathcal{C}$, make the assignment $\boldsymbol{x}[i] \leftarrow \ell_{\mathcal{C}}$, we thus construct a string on regular letters equivalent to $\mathcal{G}$; that is, a string of scope 1.

We remark that constructing the graph $\mathcal{G}$ described in Lemma 1, then checking whether or not it is a collection of cliques, is a trivial and obvious approach to determining the regularity of $\boldsymbol{x}$. In this paper we propose more efficient and interesting algorithms.

The following result is an easy corollary of Lemma 1

Corollary 1. Given a regular string $\boldsymbol{x}[1 . . n]$, then, corresponding to every triple $\left(i, j_{1}, j_{2}\right)$, we can assign a regular letter to $\boldsymbol{y}[i], \boldsymbol{y}\left[j_{1}\right], \boldsymbol{y}\left[j_{2}\right]$ in such a way that the resulting string $\boldsymbol{y}[1 . . n]$ is isomorphic to $\boldsymbol{x}[1 . . n]$.

For example, $\boldsymbol{y}=c c d c d d$ is isomorphic to $\boldsymbol{x}=a\{a, c\} b\{a, d\} b b$.

\subsection{A Model for the Representation of Indeterminate Strings}

The preceding result suggests a need for an algorithm to determine whether or not an apparently indeterminate string is nevertheless actually regular, even though, like the string $\boldsymbol{x}=a\{a, c\} b\{a, d\} b b$ mentioned in the Introduction, it contains indeterminate letters. We describe such an algorithm in the next section, but first we must deal with the following issue: in order to design algorithms on indeterminate strings $\boldsymbol{x}$, we need to have some practical representation of such strings - in particular, of indeterminate letters. This requires us to deal in some realistic way with the fact that, on an alphabet of size $\sigma$, there are $2^{\sigma}-1$ possible nonempty distinct letters. For $\sigma=2$, we have only $a, b,\{a, b\}$, but for $\sigma=26$, dealing efficiently with $2^{26}-1$ distinct letter sets is beyond current processing capabilities. Fortunately, in practice, such cases do not arise: there may be "many" letter sets but, for large $\sigma$, not $2^{\sigma}-1$ of them. 
Thus we need a model of computation that deals efficiently with indeterminate letters even when their number is large - though not "too large". For given $\sigma$, we shall accordingly assume an integer alphabet with letters $\ell \in\{1,2, \ldots, \sigma\}$ and we shall interpret letter $\ell=0$ as denoting a hole or don't-care. Otherwise, $\ell>\sigma$ represents an indeterminate letter, that also determines the position $\sigma^{\prime}=\ell-\sigma$ in an array $I\left[1 . . \sigma^{*}\right]$, where $\sigma^{*}$ is the maximum number of indeterminate letters (in addition to the don't care) allowed to occur in $\boldsymbol{x}$. Each entry $I\left[\sigma^{\prime}\right]$ is a pair $(s(\ell), l o c)$ that gives the scope $s(\ell)$ of the indeterminate letter $\ell$ and the starting location $l o c$ in an array $L$, consisting of integers in the range $\{1 \ldots \sigma\}$, of the subarray specifying the $s(\ell)$ regular letters that constitute $\boldsymbol{x}[i]$ :

$$
\left(\ell_{1}, \ell_{2}, \ldots, \ell_{s(\ell)}\right)
$$

Here the $\ell_{j}, 1 \leq j \leq s(\ell)$, are distinct elements of $\Sigma$ in normal form, thus satisfying $\ell_{1}<\ell_{2}<\cdots<\ell_{s(\ell)}$. For instance, consider $\boldsymbol{x}=$ aac $\{a, c\} \operatorname{ta}\{g, t\}\{a, c\}\{g, t\}$ over $\Sigma=\{a, c, g, t\}$. To represent it using the above encoding, we map $a \rightarrow 1, c \rightarrow 2, g \rightarrow 3, t \rightarrow 4$. Since we have two indeterminate letters in $\boldsymbol{x}, \sigma^{*}=2,0 \leq \ell \leq 6$, and $\{a, c\} \rightarrow 5$ and $\{g, t\} \rightarrow 6$. Further, $I=[(2,1),(2,3)], L=[1,2,3,4]$, and the encoding of $\boldsymbol{x}$ is 1125341656 .

To fix the ideas, we suppose here that $\ell$, hence every entry in array $L$, is constrained to one byte ( 8 bits) of storage. Thus, for example, to handle English text, we should suppose that $\sigma=77$ (26 lower case letters, 26 upper case, 10 numerical digits, 14 punctuation symbols, and space), leaving available values $\ell \in 78 . .255: \sigma^{*}=178$ distinct indeterminate letters. (Doubling storage for $\ell$ to two bytes permits 65,458 indeterminate English letters to be defined.)

Another example: for DNA strings on $\Sigma=\{a, c, g, t\}, \sigma=4$, we could define $a \rightarrow 1, c \rightarrow 2, g \rightarrow 3, t \rightarrow 4$, use zero for the don't care and 5-14 for the other 10 possible combinations of letters:

$$
a c, a g, a t, c g, c t, g t, a c g, a c t, \text { agt, cgt. }
$$


Then for DNA a half byte ( 4 bits) for $\ell$ would be sufficient to identify all the indeterminate letters, and the associated array $L$ would require at most 24 half bytes to represent them.

Other models to represent indeterminate strings have been proposed. For example, in 24] all the non-empty letters (both regular and indeterminate) over the DNA alphabet $\Sigma_{D N A}=\{A, C, G, T\}$ are mapped to the IUPAC symbols $\Sigma_{I U P A C}=\{A, C, G, T, R, Y, S, W, K, M, B, D, H, V, N\}$, to construct an isomorphic regular string of scope 1 over $\Sigma_{I U P A C}$. Further, in [25] each symbol in the DNA alphabet is represented as a 4-bit integer power of $2\left(2^{i}\right.$, with $i \in\{0,1,2,3\})$. A non-empty indeterminate letter over $\Sigma_{D N A}$ is represented as $\Sigma_{\{s \in \mathcal{P}(\Sigma)\}} s$. Then instead of using the natural order on integers it uses a Gray code [26] (also known as the reflected binary code) to order indeterminate letters over $\Sigma_{D N A}$. Note that with the Gray code two successive values differ only by one bit, such as 1100 and 1101, which enables minimizing the number of separate intervals associated with each of the four symbols of $\Sigma_{D N A}$. The models described here are of interest primarily for representing indeterminate strings over the DNA alphabet. However, the same approach is applicable to any alphabet.

\section{Determining the regularity of $x$}

In this section we discuss a function REGULAR (Figure 1) that determines whether or not a given string $\boldsymbol{x}$ is regular. REGULAR deals with strings $\boldsymbol{x}$ of scope $S(\boldsymbol{x})>1$, since otherwise, for $S(\boldsymbol{x})=1$, the regularity of $\boldsymbol{x}$ is immediate. As a byproduct, in the case that $\boldsymbol{x}$ is regular, REGULAR computes the lex-least string $\boldsymbol{y}$ of scope 1 on an integer alphabet that is isomorphic to $\boldsymbol{x}$.

Before getting into the details of REGULAR, we need to consider the overall strategy. An issue that arises is this: in order to determine whether or not $\boldsymbol{x}[1 . . n]$ is regular, it is necessary to look at all triples to determine whether or not one of them is intransitive, a process that it seems must require $\left(\begin{array}{l}n \\ 2\end{array}\right)$ letter comparisons, thus $\mathcal{O}\left(S(\boldsymbol{x}) n^{2}\right)$ time. However, it suffices to apply REGULAR 
to a reduced string $\boldsymbol{x}_{\boldsymbol{R}}=\boldsymbol{x}_{\boldsymbol{R}}[1 . . m]$ consisting of exactly one occurrence of each of the $m$ distinct letters (both regular and indeterminate) in $\boldsymbol{x}$. Thus, in view of our constraint on $\ell, m \leq 256$. Clearly $\boldsymbol{x}_{\boldsymbol{R}}$ is regular if and only if $\boldsymbol{x}$ is regular. Consequently triple-testing time reduces to $\mathcal{O}\left(m^{2}\right)$, where of course $m<<n$. (Indeed, even for the extreme two-byte case mentioned above, with $m=65,536, m^{2}$ is only 4 billion or so, thus still $\mathcal{O}(n)$ in many cases.)

function $R E G U L A R\left(\boldsymbol{x}, n ; \boldsymbol{y}, \sigma^{\prime}\right)$ : Boolean construct $\boldsymbol{x}_{\boldsymbol{R}} \triangleright$ contains each distinct letter of $\boldsymbol{x}$ exactly once $m \leftarrow\left|\boldsymbol{x}_{\boldsymbol{R}}\right|$ ret $\leftarrow$ regular $_{\min }\left(\boldsymbol{x}_{\boldsymbol{R}}, m, \boldsymbol{y}_{\boldsymbol{R}}, \sigma^{\prime}\right)$

if ret then construct $\boldsymbol{y}$ from $\boldsymbol{y}_{\boldsymbol{R}}$ $R E G U L A R \leftarrow$ ret; return

Figure 1: This Boolean function determines whether $(R E G U L A R=$ true) or not $(R E G U L A R=$ false $)$ a string $\boldsymbol{x}[1 . . n]$ of scope $S(\boldsymbol{x})>1$ on alphabet $\Sigma$ is regular. In the former case, on exit the string $\boldsymbol{y}$ is the lex-least regular string of scope 1 on an integer alphabet $\Sigma^{\prime}=\left\{1,2, \ldots, \sigma^{\prime}\right\}$ that is isomorphic to $\boldsymbol{x}$.

The first step of REGULAR therefore computes $\boldsymbol{x}_{\boldsymbol{R}}$, a process performed in $\Theta(n)$ time by a single scan of $\boldsymbol{x}$, using a Boolean array found [1..256] to indicate whether $($ found $[j]=$ true $)$ or not $($ found $[j]=$ false) the letter $j=\boldsymbol{x}[i]$ has occurred previously in $\boldsymbol{x}[1 . . i-1]$. Note that in this process, the letters are placed in $\boldsymbol{x}_{\boldsymbol{R}}$ in the order of their first occurrence in $\boldsymbol{x}$. Thus:

Claim 1. In cases of practical interest - that is, when $n$ greatly exceeds $m$ the replacement of $\boldsymbol{x}=\boldsymbol{x}[1 . . n]$ by the reduced string $\boldsymbol{x}_{\boldsymbol{R}}=\boldsymbol{x}_{\boldsymbol{R}}[1 . . m]$, where $m$ is the number of distinct letters in $\boldsymbol{x}$, permits the regularity of $\boldsymbol{x}$ to be determined in $\mathcal{O}(n)$ time.

Indeed, in many cases that arise in practice, the status of $\boldsymbol{x}$ follows from conditions that are more easily evaluated. For instance, $\boldsymbol{x}$ is indeterminate if

- it contains all regular letters in $\Sigma$ and at least one indeterminate letter; or

- it contains a don't-care and at least two regular letters; or 
- it contains an indeterminate letter $\ell$ as well as any two characters of $\ell$.

Having constructed $\boldsymbol{x}_{\boldsymbol{R}}$, REGULAR executes the Boolean function regular $_{\min }$ (Figure 21), which returns true if and only if $\boldsymbol{x}_{\boldsymbol{R}}$ is regular. In that case, regular $r_{\text {min }}$ also outputs a string $\boldsymbol{y}_{\boldsymbol{R}}$ of scope 1 on an integer alphabet $\Sigma^{\prime}$ that is isomorphic to $\boldsymbol{x}_{\boldsymbol{R}}$. As explained below, it is then straightforward to compute a lex-least string $\boldsymbol{y}$ of scope 1 isomorphic to $\boldsymbol{x}$.

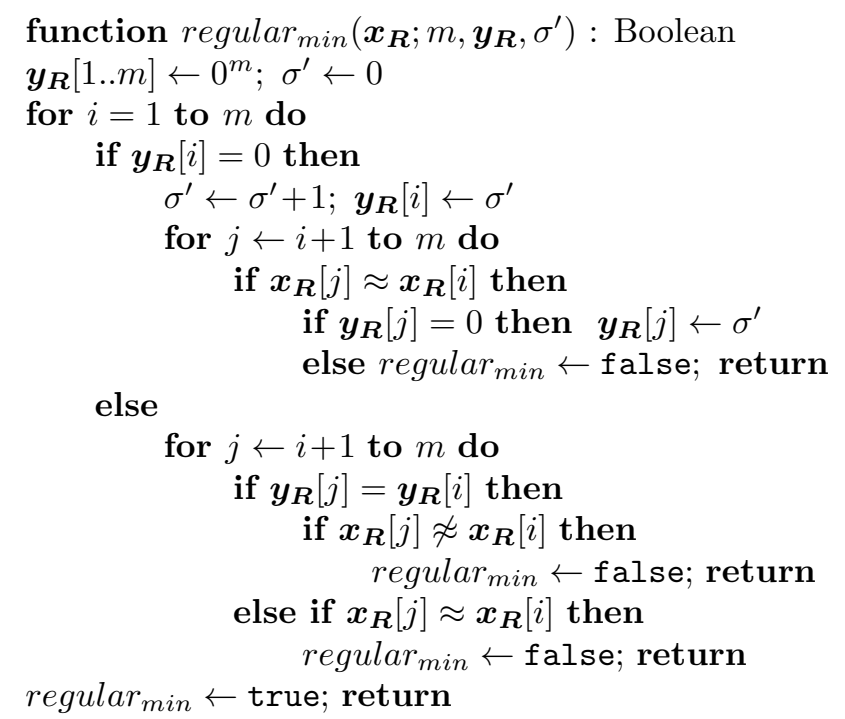

Figure 2: Determine whether $\left(\right.$ regular $_{\min }=$ true $)$ or not $\left(\right.$ regular $\left._{\min }=\mathrm{false}\right)$ a reduced string $\boldsymbol{x}_{\boldsymbol{R}}[1 . . m]$ of scope $S\left(\boldsymbol{x}_{\boldsymbol{R}}\right)>1$ on alphabet $\Sigma$ is regular. If true, on exit the string $\boldsymbol{y}_{\boldsymbol{R}}$ is the lex-least regular string of scope 1 on the integer alphabet $\Sigma^{\prime}=\left\{1,2, \ldots, \sigma^{\prime}\right\}$ that is isomorphic to $\boldsymbol{x}_{\boldsymbol{R}}$.

In the function regular ${ }_{\text {min }}$, we first initialize each letter in $\boldsymbol{y}_{\boldsymbol{R}}[1 . . m]$ to 0 . Then we scan $\boldsymbol{x}_{\boldsymbol{R}}$ from left to right, using $\boldsymbol{y}_{\boldsymbol{R}}$ to record previous matches. During this scan the following condition holds as long as $\boldsymbol{x}_{\boldsymbol{R}}$ is regular:

$$
\boldsymbol{x}_{\boldsymbol{R}}[i] \approx \boldsymbol{x}_{\boldsymbol{R}}[j] \Leftrightarrow \boldsymbol{y}_{\boldsymbol{R}}[i]=\boldsymbol{y}_{\boldsymbol{R}}[j] \wedge \boldsymbol{y}_{\boldsymbol{R}}[i] \neq 0 .
$$

If at position $i \in\{1, \ldots, m\}$, we find $\boldsymbol{y}_{\boldsymbol{R}}[i]=0$ - that is, it was not part of a previous match - we form the new character $\sigma^{\prime} \leftarrow \sigma^{\prime}+1$. We then scan right in the strings $\boldsymbol{x}_{\boldsymbol{R}}[i+1 . . m]$ and $\boldsymbol{y}_{\boldsymbol{R}}[i+1 . . m]$ to see if condition (2) continues to hold. If it does not, we mark $\boldsymbol{x}_{\boldsymbol{R}}$ as indeterminate and exit; otherwise, whenever 
$\boldsymbol{x}_{\boldsymbol{R}}[j] \approx \boldsymbol{x}_{\boldsymbol{R}}[i]$ and $\boldsymbol{y}_{\boldsymbol{R}}[j]=0$, we assign $\boldsymbol{y}_{\boldsymbol{R}}[j] \leftarrow \sigma^{\prime}$.

In regular ${ }_{\text {min }}$ the inner loops execute $\left(\begin{array}{c}m \\ 2\end{array}\right)$ times, and checking if two letters match takes $\mathcal{O}(\sigma)$ time for $\boldsymbol{x}$ in normal form, $\mathcal{O}(\sigma \log \sigma)$ otherwise. Therefore:

Lemma 2. Algorithm regular ${ }_{\text {min }}$ runs in $\mathcal{O}\left(m^{2} \sigma\right)$ time, where $m=\left|\boldsymbol{x}_{\boldsymbol{R}}\right|$ and $1 \leq m \leq 2^{\sigma}$, when $\boldsymbol{x}_{\boldsymbol{R}}$ is in normal form; otherwise, $\mathcal{O}\left(m^{2} \sigma \log \sigma\right)$ time, where $\mathcal{O}(\sigma \log \sigma)$ is the maximum time needed to sort the characters in two letters.

Lemma 3. When $\boldsymbol{x}[1 . . n]$ is regular, the (lex-least) corresponding $\boldsymbol{y}$ can be computed from $\boldsymbol{y}_{\boldsymbol{R}}$ in $\Theta(n)$ time.

Proof. As noted above, the letters in $\boldsymbol{x}_{\boldsymbol{R}}[1 . . m]$ occur in order of their first appearance in $\boldsymbol{x}$. Moreover, the letters in $\boldsymbol{y}_{\boldsymbol{R}}$ are assigned in increasing order $1,2, \ldots, \sigma^{\prime}$ of occurrence of the positions of new letters in $\boldsymbol{x}_{\boldsymbol{R}}$. Thus, to compute $\boldsymbol{y}$, we first form $P_{\boldsymbol{x}_{\boldsymbol{R}}}[1 . . n]$, in which $P_{\boldsymbol{x}_{\boldsymbol{R}}}[j]$ is the position at which the letter $\boldsymbol{x}[j]$ occurs in $\boldsymbol{x}_{\boldsymbol{R}}$. Therefore, the elements of $\boldsymbol{P}_{\boldsymbol{x}_{\boldsymbol{R}}}$ are integers in the range $\{1 \ldots m\}$. Clearly, $P_{\boldsymbol{x}_{\boldsymbol{R}}}[1 . . n]$ can be computed in $\Theta(n)$ time. Then for every $i \in\{1 \ldots n\}$, we compute

$$
\boldsymbol{y}[i] \leftarrow \boldsymbol{y}_{\boldsymbol{R}}\left[P \boldsymbol{x}_{\boldsymbol{R}}[i]\right],
$$

yielding a lex-least string of scope 1 isomorphic to $\boldsymbol{x}$.

Lemma 4. Algorithm REGULAR runs in $\Theta(n)$ time for a string $\boldsymbol{x}[1 . . n]$ from a constant alphabet.

Proof. The running time of REGULAR is equal to the total time required for (1) construction of $\boldsymbol{x}_{\boldsymbol{R}}$ from $\boldsymbol{x}$; (2) execution of regular $_{\text {min }}$; (3) construction of $\boldsymbol{y}$ from $\boldsymbol{y}_{\boldsymbol{R}}$.

As we have seen (Claim 1), (1) requires $\Theta(n)$ time, while Lemma 2 tells us that (2) requires $\mathcal{O}\left(m^{2} \sigma \log \sigma\right)$ time. However, since $m$ and $\sigma$ are constants, (2) requires constant time. Further, as explained in Lemma $3 \boldsymbol{y}$ can be computed from $\boldsymbol{y}_{\boldsymbol{R}}$ in $\Theta(n)$ time. Therefore, we conclude that the running time of 
REGULAR is $\Theta(n)$.

At a cost of adding $\Theta\left(m^{2}\right)$ bits of additional storage, together with $\mathcal{O}\left(m^{2} \sigma\right)$ preprocessing time, the regularity of the reduced string $\boldsymbol{x}_{\boldsymbol{R}}$ can actually be determined in $\mathcal{O}\left(\mathrm{m}^{2}\right)$ time in all cases, as we now explain. Essentially, we replace $\boldsymbol{x}_{\boldsymbol{R}}$ by a symmetric adjacency matrix $M_{\widetilde{\boldsymbol{x}}_{\boldsymbol{R}}}=M_{\widetilde{\boldsymbol{x}}_{\boldsymbol{R}}}[1 . . m, 1 . . m]$ based on the matches in $\boldsymbol{x}_{\boldsymbol{R}}$ : for $1 \leq i, j \leq m$,

$$
M \widetilde{\boldsymbol{x}}_{\boldsymbol{R}}[i, j]= \begin{cases}1 & \text { if } \boldsymbol{x}_{\boldsymbol{R}}[i] \approx \boldsymbol{x}_{\boldsymbol{R}}[j] \\ 0 & \text { otherwise }\end{cases}
$$

Since each match may require $\mathcal{O}(\sigma)$ time, this construction can be performed in $\mathcal{O}\left(m^{2} \sigma\right)$ time in the worst case.

Then, simply replacing references to $\boldsymbol{x}_{\boldsymbol{R}}$ in function regular_min by references to $M \widetilde{\widetilde{\boldsymbol{x}}}_{\boldsymbol{R}}$, we obtain the $\mathcal{O}\left(m^{2}\right)$ function regular_min_matrix (Figure 3).

$$
\begin{aligned}
& \text { function regular min_matrix }\left(M \widetilde{\boldsymbol{x}}_{\boldsymbol{R}}, m ; \boldsymbol{y}_{\boldsymbol{R}}, \sigma^{\prime}\right) \\
& \boldsymbol{y}_{\boldsymbol{R}}[1 . . m] \leftarrow 0^{m} ; \sigma^{\prime} \leftarrow 0 \\
& \text { for } i=1 \text { to } m \text { do } \\
& \text { if } \boldsymbol{y}_{\boldsymbol{R}}[i]=0 \text { then } \\
& \sigma^{\prime} \leftarrow \sigma^{\prime}+1 ; \boldsymbol{y}_{\boldsymbol{R}}[i] \leftarrow \sigma^{\prime} \\
& \text { for } j \leftarrow i+1 \text { to } m \text { do } \\
& \text { if } M_{\widetilde{\boldsymbol{x}}_{\boldsymbol{R}}}[i, j]=1 \text { then } \\
& \text { if } \boldsymbol{y}_{\boldsymbol{R}}[j]=0 \text { then } \boldsymbol{y}_{\boldsymbol{R}}[j] \leftarrow \sigma^{\prime} \\
& \text { else } \text { regular }_{\text {min_matrix }} \leftarrow \text { false; return } \\
& \text { for } j \leftarrow i+1 \text { to } m \text { do } \\
& \text { if } \boldsymbol{y}_{\boldsymbol{R}}[j]=\boldsymbol{y}_{\boldsymbol{R}}[i] \text { then } \\
& \text { if } M_{\widetilde{\boldsymbol{x}}_{\boldsymbol{R}}}[i, j]=0 \text { then } \\
& \text { regular }_{\text {min_matrix }} \leftarrow \text { false; return } \\
& \text { else if } M_{\widetilde{\boldsymbol{x}}_{\boldsymbol{R}}}[i, j]=1 \text { then } \\
& \text { regular }_{\text {min_matrix }} \leftarrow \text { false; return } \\
& \text { regular }_{\text {min_matrix }} \leftarrow \text { true; return }
\end{aligned}
$$

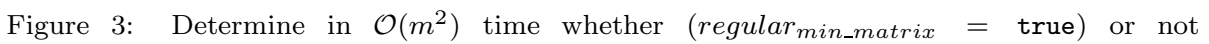
$\left(\right.$ regular $_{\text {min_matrix }}=$ false $)$ an $m \times m$ bit matrix $M_{\widetilde{\boldsymbol{x}}_{\boldsymbol{R}}}$ is regular. In the former case, the algorithm computes, corresponding to $M_{\widetilde{\boldsymbol{x}}_{\boldsymbol{R}}}$, a lex-least regular string $\boldsymbol{y}_{\boldsymbol{R}}$ of scope 1 on the integer alphabet $\Sigma^{\prime}=\left\{1,2, \ldots, \sigma^{\prime}\right\}$.

Effectively, $M \widetilde{\boldsymbol{x}}_{\boldsymbol{R}}$ defines an undirected graph $G \widetilde{\widetilde{\boldsymbol{x}}}_{\boldsymbol{R}}=(V, E)$ on $m$ ver- 
tices, where there exists an edge $(i, j) \in E$ if and only if $M_{\widetilde{\boldsymbol{x}}_{\boldsymbol{R}}}[i, j]=1$. The match relation $\approx$ is necessarily both reflexive $\left(M_{\widetilde{\boldsymbol{w}}}[i, i]=1\right)$ and symmetric $(M \widetilde{\widetilde{\boldsymbol{w}}}[i, j]=M \widetilde{\widetilde{\boldsymbol{w}}}[j, i])$ for any string $\boldsymbol{w}$. If in addition our string $\boldsymbol{w}=\boldsymbol{x}_{\boldsymbol{R}}$ is regular, it follows immediately from our definition that the match relation is transitive:

$$
M \widetilde{\widetilde{\boldsymbol{x}}}_{\boldsymbol{R}}\left[i, j_{1}\right]=M \widetilde{\widetilde{\boldsymbol{x}}}_{\boldsymbol{R}}\left[i, j_{2}\right] \Longleftrightarrow M_{\widetilde{\boldsymbol{x}}_{\boldsymbol{R}}}\left[j_{1}, j_{2}\right]=M_{\widetilde{\boldsymbol{x}}_{\boldsymbol{R}}}\left[i, j_{1}\right]
$$

Thus for a regular string $\boldsymbol{x}_{\boldsymbol{R}}$, algorithms regular $_{\text {min }}$ and regular $r_{\text {min_matrix }}$ effectively compute the transitive closure of the match relation $\approx$ on $\boldsymbol{x}_{\boldsymbol{R}}$ (equivalently, of the adjacent vertices in $G \widetilde{\widetilde{x}}_{R}$ ); that is, they identify the maximum sets of positions that match in $\boldsymbol{x}_{\boldsymbol{R}}$ (the cliques in $G \widetilde{\widetilde{\boldsymbol{x}}}_{\boldsymbol{R}}$ ).

In the literature the Floyd-Warshall algorithms [27, 28] are the standard for computing the transitive closure on a graph $G=(V, E)$, with $|V|=m$. These algorithms execute in time $\mathcal{O}\left(\mathrm{m}^{3}\right)$, and it is a long-standing open problem whether a faster algorithm exists. They are very general, not assuming the reflexive and symmetric conditions that hold in our case. Nevertheless, we have:

Lemma 5. For a relation on $m$ objects that is reflexive and symmetric, the transitive closure can be computed in $\mathcal{O}\left(\mathrm{m}^{2}\right)$ time.

\section{Strings from the Maximal Palindrome Array}

Given $\boldsymbol{x}=\boldsymbol{x}[1 . . n]$, a substring $\boldsymbol{u}=\boldsymbol{x}[i . . j], 1 \leq i \leq j \leq n$, of length $\ell=j-i+1$ is said to be a palindrome if $\boldsymbol{x}[i+h] \approx \boldsymbol{x}[j-h]$ for every $h \in 0 . .\lfloor\ell / 2\rfloor$; a maximal palindrome if one of the following holds: $i=1, j=n$, or $\boldsymbol{x}[i-1] \not \approx$ $\boldsymbol{x}[j+1]$. The centre of a palindrome $\boldsymbol{u}$ is at position $i+\frac{\ell-1}{2}$, which is not an integer for odd $\ell$. Thus to facilitate discussion of the palindromic structure of $\boldsymbol{x}$, we form the string

$$
\boldsymbol{x}^{*}[1 . . m]=\# x_{1} \# x_{2} \# \cdots \# x_{n} \#
$$


where $\# \notin \Sigma$ and $m=2 n+1$. Now every palindromic substring of $\boldsymbol{x}$ of length $\ell \geq 1$ maps into a palindromic substring of $x^{*}$ of odd length $d=2 \ell+1$, and every palindrome in $\boldsymbol{x}^{*}$ has an integral centre position. We call $d$ the diameter and $r=\lfloor d / 2\rfloor$ the radius of the palindrome in $\boldsymbol{x}^{*}$.

We can now define the maximal palindrome array MP $=\mathrm{MP}_{\boldsymbol{x}^{*}}$ of $\boldsymbol{x}^{*}$ : for every $i \in\{1, \ldots, m\}$, if $\boldsymbol{x}^{*}[i]=\#$ and $\boldsymbol{x}^{*}[i-1] \not \approx \boldsymbol{x}^{*}[i+1]$, then $\operatorname{MP}[i]=0$ (radius zero); otherwise, $\operatorname{MP}[i] \geq 1$ is the radius of the maximal palindrome centred at position $i$. We assume that $\boldsymbol{x}^{*}[0]=\boldsymbol{x}^{*}[m+1]=\emptyset$. For example, $\mathrm{MP}_{\boldsymbol{x}^{*}}$ derived from $\boldsymbol{x}=$ aabac is as follows:

$\begin{array}{rrrrrrrrrrrr}1 & 2 & 3 & 4 & 5 & 6 & 7 & 8 & 9 & 10 & 11 \\ \boldsymbol{x}^{*}=\# & a & \# & a & \# & b & \# & a & \# & c & \# \\ \mathrm{MP}_{\boldsymbol{x}^{*}=0} & 1 & 2 & 1 & 0 & 3 & 0 & 1 & 0 & 1 & 0\end{array}$

The most general form of the palindrome array is given by

$$
\mathrm{MP}=0 i_{2} i_{3} \cdots i_{m-1} 0
$$

where for every $j \in\{2, \ldots, m-1\}$ :

(a) $i_{j} \in(1-j \bmod 2) . . \min (j-1, m-j)$;

(b) $i_{j}$ is odd if and only if $j$ is even.

Any array satisfying (5) is said to be feasible.

Lemma 6. There exists a string corresponding to every feasible palindrome array.

Proof. Suppose that a feasible palindrome array MP $=\mathrm{MP}_{\boldsymbol{x}^{*}}[1 . . m]$ is given for some odd positive integer $m$. We show how to construct a corresponding $\boldsymbol{x}^{*}$. First, for every odd $c \in\{1, \ldots, m\}$, we may of course assign $\boldsymbol{x}^{*}[c] \leftarrow \#$. Suppose that the even positions $c$ in $\boldsymbol{x}^{*}$ are initially empty. For every $c \in\{3, \ldots, m-2\}$ such that $\operatorname{MP}[i]=r \geq 2$, add a unique character to each pair of positions $c-k, c+k$ in $\boldsymbol{x}^{*}$, where 
- ( $c$ even, $r$ odd) $k=2,4, \ldots, r-1$;

- ( $c$ odd, $r$ even) $k=1,3, \ldots, r-1$.

Finally, assign a unique character to each position that remains empty. The resulting (perhaps indeterminate) string will have MP as its palindrome array.

Applying the construction of Lemma 6 to $\mathrm{MP}_{\boldsymbol{x}^{*}}=01210301010$ given in (4) yields a string such as:

$\begin{array}{rrccccccccc}1 & 2 & 3 & 4 & 5 & 6 & 7 & 8 & 9 & 10 & 11 \\ \boldsymbol{x}^{*}=\# & a & \# & \{a, b\} & \# & c & \# & b & \# & d & \# \\ \mathrm{MP}_{\boldsymbol{x}^{*}}=0 & 1 & 2 & 1 & 0 & 3 & 0 & 1 & 0 & 1 & 0\end{array}$

Note that, since the triple $(4,2,8)$ is intransitive $(\boldsymbol{x}[2] \not \approx \boldsymbol{x}[8]), \boldsymbol{x}^{*}$ is indeterminate. However, as we have seen in (4), the regular string \#a\#a\#b\#a\#c\# has the same palindrome array: a palindrome array can correspond to both a regular and an indeterminate string!

To each position $c \in\{1, \ldots, m\}$ of a feasible MP array we associate a pair of integers $(i, j)$ such that $i=c-\operatorname{MP}[c]-1$ and $j=c+\mathrm{MP}[c]+1$; then, provided $0<i, j<m+1$, we must have $\boldsymbol{x}^{*}[i] \not \approx \boldsymbol{x}^{*}[j]$. We call this pair $(i, j)$ the forbidden pair with respect to $c$, and the characters at $\boldsymbol{x}^{*}[i], \boldsymbol{x}^{*}[j]$ forbidden characters with respect to $c$. For processing purposes, it will be convenient to assume that $\boldsymbol{x}^{*}[0]=\boldsymbol{x}^{*}[m+1]=\emptyset$. We denote by FP the set of all forbidden pairs with respect to each centre $c \in\{1, \ldots, m\}$. See (7) for an example.

Consider the feasible MP array given in (7). This array does not correspond to any regular string because the triple $(6,2,4)$ is intransitive $\left(\boldsymbol{x}^{*}[2] \not \approx \boldsymbol{x}^{*}[4]\right)$, since the pair $(2,4)$ is a forbidden pair with respect to the centre 3 . Indeed, any triple of $\boldsymbol{x}^{*}$ which includes a forbidden pair is intransitive, resulting in an indeterminate string. However, if a given MP array does correspond to a regular 
string, we will say that it is regular.

$\begin{array}{rrrccccc}c & 1 & 2 & 3 & 4 & 5 & 6 & 7 \\ M P & 0 & 1 & 0 & 3 & 2 & 1 & 0 \\ \boldsymbol{x}^{*} & \# & 1 & \# & \{2,3\} & \# & \{1,3\} & \# \\ \mathrm{FP} & (0,2) & (0,4) & (2,4) & (0,8) & (2,8) & (4,8) & (6,8)\end{array}$

In order to characterize MP arrays, it is useful to introduce Manacher's condition [29], restated in [23], and further restated here.

In MP $=\mathrm{MP}_{\boldsymbol{x}^{*}}$, we consider each centre $c$ of a palindrome of radius $r=$ $\mathrm{MP}[c]$, where for $c$ even, $r \geq 3$, and for $c$ odd, $r \geq 2$. Then we must have $\boldsymbol{x}^{*}[c-k] \approx \boldsymbol{x}^{*}[c+k]$, where $1 \leq k \leq r$. We then have

Definition 1. [Manacher's condition] Let $r_{\ell}=M P[c-k]$ and $r_{r}=M P[c+k]$, for each $1 \leq k \leq r$, where $r=M P[c]$. Every position $c$ in a regular palindrome array $M P=M P_{\boldsymbol{x}^{*}}$ satisfies the following:

(a) if $r_{\ell} \neq r-k$ then $r_{r}=\min \left(r_{\ell}, r-k\right)$ else $r_{r} \geq r_{\ell}$;

(b) if $r_{r} \neq r-k$ then $r_{\ell}=\min \left(r_{r}, r-k\right)$ else $r_{\ell} \geq r_{r}$.

For completeness, we outline here Manacher's algorithm to construct an MP array for a given string $\boldsymbol{x}^{*}$ of scope 1 . The algorithm scans $\boldsymbol{x}^{*}$ from left to right and evaluates centres to compute $\mathrm{MP}[c]$. While evaluating a centre $c$ and each value of the range $k$, it checks whether $r_{\ell}=r-k$; if so, it stops evaluating centre $c$ and moves to evaluating centre $c+k$; otherwise it assigns $r_{r} \leftarrow \min \left(r_{\ell}, r-k\right)$ and continues evaluating the same centre $c$ and the next range value $k+1$.

In 29] it is shown that, for every palindrome in string $\boldsymbol{x}^{*}$ such that $S\left(\boldsymbol{x}^{*}\right)=$ 1, Manacher's condition must hold. Thus, by Lemma 1, Manacher's condition holds for every regular string; that is, for every string whose triples are all transitive.

On the other hand, note that in example (7), for $c=4$ we have

$$
r=\mathrm{MP}[4]=3, k=1, r_{\ell}=\mathrm{MP}[3]=0, r_{r}=\mathrm{MP}[5]=2,
$$


so that, by Definition 1(a), since $r_{\ell} \neq r-k$, therefore $r_{r}=\min (0,2)=0$ should hold, which is false. Thus for this indeterminate string, Manacher's condition does not hold.

The above suggests extending the construction of Lemma [ so as to yield, whenever possible, a regular string corresponding to a given palindrome array. See Figure 4

The procedure construct takes a feasible MP array as input and returns a lex-least regular string $\boldsymbol{x}^{*}$ if MP is regular; otherwise it returns an indeterminate string. This procedure is based on the Manacher's algorithm presented in [29]. To ensure that the resulting string, if regular, is a lex-least string, it maintains for each centre $c$ a set $\mathrm{FS}[c]$ containing the indices of the forbidden characters.

In procedure construct the output string is first initialized with 0 at even positions and \# at odd positions. Then $\boldsymbol{x}^{*}[2]$ is set to 1 , as we are interested in the lex-least regular $\boldsymbol{x}^{*}$, if it exists. Next we examine a centre $3 \leq c \leq m-1$ and update the FS $[c]$ set based on its forbidden pair. If the string $\boldsymbol{x}^{*}[1 . . c-1]$ constructed so far is regular and the centre $c$ and range $k$ satisfy Manacher's condition, we continue to construct the regular string by copying the previously filled letter $\boldsymbol{x}^{*}[c-k]$ to its corresponding matching position $c+k$. Whenever there is a choice of filling an empty position - that is, when $x^{*}[c]=0$ - the lex-least character which is not in the forbidden set of characters $\mathrm{FS}[c]$ is chosen. Note that we use the same strategy as presented in Manacher's algorithm [29] to avoid evaluating every centre if MP is regular. However, unlike Manacher's algorithm, instead of evaluating a new centre when $r_{\ell}=r-k$ and $r_{r}>r_{\ell}$, we record the next centre to be evaluated, and finish evaluating the current centre. After which we move to the recorded centre and check if Manacher's condition is satisfied.

Furthermore, to avoid invoking the updateFS procedure repeatedly on a centre $c$, we use a Boolean array empty of length $m$, such that empty $[c]=$ true if and only if the centre $c$ has not been used to update the FS array; otherwise, we set empty $[c] \leftarrow$ false. If a given centre $c$ and range $k$ do not satisfy Manacher's condition, regular is set to false and every subsequent letter match including 
function construct(MP, $m ; \boldsymbol{x}^{*}, \sigma$, regular)

$\triangleright M P[1 . . m]$ is assumed to be feasible, and $m=\left|\boldsymbol{x}^{*}\right|$.

$\triangleright F S[1 . . m]$ gives the index position of all forbidden characters w.r.t each centre of the MP array.

$\triangleright$ empty $[1 . . m]$ is a Boolean array initially set to true, where

$\triangleright$ empty $[c]=$ true whenever updateFS has not been called for centre $c$. regular $\leftarrow$ true; $\sigma \leftarrow 0 ; \boldsymbol{x}^{*}[1] \leftarrow \# ; \mathrm{FS}[1] \leftarrow \emptyset$

for $c \leftarrow 2$ to $m-1$ step 2 do $\quad \triangleright$ Initialize $\boldsymbol{x}^{*}$ and FS array.

$\boldsymbol{x}^{*}[c] \leftarrow 0 ; \mathrm{FS}[c] \leftarrow \emptyset$

$\boldsymbol{x}^{*}[c+1] \leftarrow \# ; \mathrm{FS}[c+1] \leftarrow \emptyset$

empty $[1 . .2]=$ false; empty $[3 . . m]=$ true

$\sigma \leftarrow \sigma+1 ; \boldsymbol{x}^{*}[2] \leftarrow \sigma$

for $c \leftarrow 3$ to $m-1$

$r \leftarrow \mathrm{MP}[c]$

if regular and empty $[c]$ then

empty $[c] \leftarrow$ false; update $\mathrm{FS}(c, r, m, \mathrm{FS})$

if $r>1$ then

nextc $\leftarrow c+r+1$

for $k \leftarrow r$ downto 1 do

$r_{\ell}=\operatorname{MP}[c-k] ; r_{r}=\operatorname{MP}[c+k]$

$\triangleright M C$ is a function that tests Manacher's condition

$\triangleright$ for centre $c$, radius $r$ and range $k$.

if regular and $M C(c, r, k)$ then

if empty $[c+k]$ then

empty $[c+k] \leftarrow$ false; update $\mathrm{FS}\left(c+k, r_{r}, m, \mathrm{FS}\right)$

if $\boldsymbol{x}^{*}[c+k]=0$ then $\boldsymbol{x}^{*}[c+k] \leftarrow \boldsymbol{x}^{*}[c-k]$

if $r_{\ell}=r-k$ and $r_{r}>r_{\ell}$ then nextc $\leftarrow c+k$

else

regular $\leftarrow$ false

if $c+k \bmod 2=0$ and $\boldsymbol{x}^{*}[c-k] \not \approx \boldsymbol{x}^{*}[c+k]$ then

$k \leftarrow k-1$

$\sigma \leftarrow \sigma+1 ; \boldsymbol{x}^{*}[c-k] \stackrel{+}{\leftarrow} \sigma ; \boldsymbol{x}^{*}[c+k] \stackrel{+}{\leftarrow} \sigma$
if $\boldsymbol{x}^{*}[c]=0$ then
if regular then
else $\sigma \leftarrow \sigma+1 ; \boldsymbol{x}^{*}[c] \stackrel{+}{\leftarrow} \sigma$
if regular then $c \leftarrow$ nextc
else $c \leftarrow c+1$
return $x^{*}$

$\boldsymbol{x}^{*}[c] \leftarrow$ Lex-least character not in $\mathrm{FS}[c]$

Figure 4: Given a feasible palindrome array MP, construct if possible produces a corresponding lex-least regular string $\boldsymbol{x}^{*}$ of scope 1 on an integer alphabet $\Sigma=\{1,2, \ldots\}$. If such a string is not possible, then on exit regular $=$ false and $\boldsymbol{x}^{*}$ is an indeterminate string on $\Sigma$ corresponding to MP. 


$$
\begin{aligned}
& \text { procedure update } \mathrm{FS}(c, r, m, \mathrm{FS}) \\
& \text { if } c-r-1 \neq 0 \text { and } c+r+1 \neq m+1 \text { then } \\
& \qquad \mathrm{FS}[c+r+1] \stackrel{+}{\leftarrow} c-r-1
\end{aligned}
$$

Figure 5: Given centre $c$ and its radius $r$, updateFS updates the FS array at the left position in the forbidden pair with respect to $c$.

the current one is achieved by adding a new character to the letters at positions $c-k$ and $c+k$, if they are even. Furthermore, for the remainder of the MP array all centres are examined.

Lemma 7. Let $\boldsymbol{x}^{*}$ be the string produced by procedure construct. Then $S\left(\boldsymbol{x}^{*}\right)=1 \Leftrightarrow \boldsymbol{x}^{*}$ is regular.

Proof. $\quad(\Rightarrow)$ Since $S\left(x^{*}\right)=1$, the regularity of $\boldsymbol{x}^{*}$ is immediate, as for all positions $1 \leq i, j \leq m, \boldsymbol{x}^{*}[i] \approx \boldsymbol{x}^{*}[j] \Leftrightarrow \boldsymbol{x}^{*}[i]=\boldsymbol{x}^{*}[j]$. Then any triple in $\boldsymbol{x}^{*}$ is transitive by the associative property of equality. Therefore $\boldsymbol{x}^{*}$ is regular.

$(\Leftarrow) x^{*}$ is regular only if Manacher's condition holds for every centre $c$, radius $r$ and range $k$. In such a case construct either copies existing letters of scope one or fills an empty position with a new character to produce $\boldsymbol{x}^{*}$. Therefore $S\left(\boldsymbol{x}^{*}\right)=1$.

Theorem 1. Let $\boldsymbol{x}^{*}$ be the string produced by the procedure construct on an input MP. Then $\boldsymbol{x}^{*}$ is regular $\Leftrightarrow M P$ is regular.

Proof. $(\Rightarrow) \boldsymbol{x}^{*}$ is regular only if Manacher's condition holds for every centre $c \in$ $\{1, \ldots, m\}$, and for every corresponding radius $r$ and range $k$. Since Manacher's condition holds, it follows from the result in [29] that MP is regular.

$(\Leftarrow)$ From [29], MP is regular only if Manacher's condition holds. Since the condition holds, during the execution of construct an empty position is filled either by copying previously occurring letters of scope one or by a new character. Thus the resulting string is of scope 1 , so that from Lemma $7 \boldsymbol{x}^{*}$ is regular. 
Using the two examples given below in (8) and (9), we illustrate the execution of procedure construct.

In (8) the positions corresponding to all odd centres are filled with \# and positions corresponding to all even centres are filled with 0. Furthermore, $\mathrm{FS}[1 . . m] \leftarrow \emptyset$, empty[1..2] $\leftarrow$ false and empty[3..m] $\leftarrow$ true. Then for centre $c=2, \boldsymbol{x}^{*}[2] \leftarrow 1$, and for centre $c=3, F S[4] \leftarrow\{2\}$ and empty $[3] \leftarrow$ false. At centre $c=4$, empty[4] $\leftarrow$ false, and since $r=3$ we check if Manacher's condition holds for $k=3,2,1$. As we do this, we also check whether empty $[c+k]$ is true; if so, we set it to false and compute the FS set w.r.t to these centres, thereby setting FS[6] and FS[8] to $\{4\},\{4,6\}$ respectively. Since Mancher's condition holds, the setting at position $\boldsymbol{x}^{*}[2]$ is copied into $\boldsymbol{x}^{*}[6]$; next, since position $\boldsymbol{x}^{*}[4]=0$, it is filled with the next lex-least character which is not equal to a character at any index position in the forbidden set $\operatorname{FS}[4]=\{2\}$ - that is, 2 .

Similarly for centre $c=8$, empty[8..15] $\leftarrow$ false, FS[10] $\leftarrow\{8\}$, FS[12] $\leftarrow$ $\{8,10\}$. Since Manacher's condition holds for all $k=7,6, \ldots, 1, \boldsymbol{x}^{*}[2], \boldsymbol{x}^{*}[4]$ and $\boldsymbol{x}^{*}[6]$ are copied into $\boldsymbol{x}^{*}[14], \boldsymbol{x}^{*}[12]$ and $\boldsymbol{x}^{*}[10]$, respectively. Finally, since $\boldsymbol{x}^{*}[8]=0$, the position is filled with the next lex-least character 3 which is not equal to a character at any index position in the forbidden set $\operatorname{FS}[8]=\{4,6\}$. The resulting string $\boldsymbol{x}^{*}$ is regular and lex-least.

$\begin{array}{rcccccccccccccc}1 & 2 & 3 & 4 & 5 & 6 & 7 & 8 & 9 & 10 & 11 & 12 & 13 & 14 & 15 \\ M P=0 & 1 & 0 & 3 & 0 & 1 & 0 & 7 & 0 & 1 & 0 & 3 & 0 & 1 & 0 \\ \boldsymbol{x}^{*}=\# & 1 & \# & 2 & \# & 1 & \# & 3 & \# & 1 & \# & 2 & \# & 1 & \# \\ F S=\emptyset & \emptyset & \emptyset & \{2\} & \emptyset & \{4\} & \emptyset & \{4,6\} & \emptyset & \{8\} & \emptyset & \{8,10\} & \emptyset & \{12\} & \emptyset\end{array}$

Observe that the MP array in (9) only differs from the MP array in (8) at position 12. However, the MP array in (9) does not correspond to any regular string, since, as we shall see, it fails Manacher's condition for $c=8, r=7$, and $k=4$. In (9) construct produces the same string as in (8) up until $c=7$. Then at centre $c=8, \boldsymbol{x}^{*}[2]$ is first copied into $\boldsymbol{x}^{*}[14]$. However, since for $k=4$ the MP array fails Manacher's condition, a new lex-least character 3 is added to 
the letters at positions 4 and 12. Then at positions 6 and 10, a new character 4 is added to both $\boldsymbol{x}^{*}[6]$ and $\boldsymbol{x}^{*}[10]$. Finally, since $\boldsymbol{x}^{*}[8]=0$, it is filled with another new character 5 .

$\begin{array}{rrrrrrrrrrrrrrr}1 & 2 & 3 & 4 & 5 & 6 & 7 & 8 & 9 & 10 & 11 & 12 & 13 & 14 & 15 \\ M P=0 & 1 & 0 & 3 & 0 & 1 & 0 & 7 & 0 & 1 & 0 & 1 & 0 & 1 & 0 \\ \boldsymbol{x}^{*}=\# & 1 & \# & \{2,3\} & \# & \{1,4\} & \# & 5 & \# & 4 & \# & 3 & \# & 1 & \#\end{array}$

We now discuss the running time complexity of construct. This is dependent on the input MP array. If MP is regular, then construct yields a lex-least regular string in $\mathcal{O}(n)$ time; otherwise it yields an indeterminate string in $\mathcal{O}\left(n^{2}\right)$ time. We remark that to avoid having $\sigma$ in the running time, we use the strategy discussed in 23] to fill the lex-least character not in the list of forbidden characters at centre $c$. Here we briefly outline the strategy: The paper [23] uses a bit vector $F$ of length $n$ where the element $F[h]$ corresponds to the $h$-th lex-least least character. Initially all elements of $F$ are set to 0 . If a character $\boldsymbol{x}[j]$ is forbidden w.r.t the character $\boldsymbol{x}[i]$, and if $\boldsymbol{x}[j]$ is the $h$-th lex-least character, then $F[h]$ is set to 1 . After finding all forbidden characters for $\boldsymbol{x}[i]$ and setting the $F$ vector appropriately, the lex-least character for $\boldsymbol{x}[i]$ is set equal to the $k$-th lex-least character, where $k$ is the first (or smallest) index such that $F[k]=0$.

If MP is regular, then similar to Manacher's algorithm, not every centre is evaluated to yield a regular string. Suppose $c, 1 \leq c \leq m$, is the least centre for which the Manacher's test is performed and is satisfied. Then, two cases arise. First, there exists a $k$ (in construct we consider the minimum $k$ ), $1 \leq$ $k \leq r$, where the following subcondition of Manacher's condition (Definition 1) is satisfied: $r_{\ell}=r-k$ and $r_{r}>r_{\ell}$. Second, when the subcondition is not satisfied, all the palindromes that arise at centres $c^{\prime} \leq c+r$ are contained in the palindrome at $c$ and also satisfy Manacher's condition. Therefore, to avoid duplication the next centre to be evaluated is $c+r+1$.

In the former case, however, the palindrome at centre $c+k$ extends beyond the right edge of the palindrome centred at $c$. Therefore, $c+k$ is the next centre 
to be evaluated. However, since $c$ satisfies Manacher's, a shorter palindrome of length $r-k$ at centre $c+k$ also occurs at centre $c-k$. Therefore, we only need to check the positions occurring after the first $r-k$ positions on both sides of $c+k$. In both these cases a position in the MP array is visited at most twice. Therefore, the running time of construct to yield a regular string is $\mathcal{O}(n)$.

If MP is not regular, after the first centre that fails Manacher's test, every centre is evaluated, and as a result construct yields an indeterminate string in $\mathcal{O}\left(n^{2}\right)$ time. However, note that the running time for an MP array that is not regular largely depends on the centre that fails the Manacher's test. If the centre lies close to the end of the array, then the indeterminate string is constructed in $\mathcal{O}(n)$ time; otherwise it takes $\mathcal{O}\left(n^{2}\right)$ time. Thus we have:

Theorem 2. Given a regular MP array of length $m=2 n+1$, procedure construct yields a lex-least regular string in $\mathcal{O}(n)$ time.

Theorem 3. Given a MP array of length $m=2 n+1$ that is not regular, procedure construct yields an indeterminate string in $\mathcal{O}\left(n^{2}\right)$ time.

We remark that our algorithm construct is similar to the approach presented in 23, Algorithm 2] to construct a regular string if MP is regular. The key differences between construct and their approach is that their algorithm sometimes verifies an MP array which is not regular to be regular, and therefore outputs an incorrect lex-least regular string $\boldsymbol{w}$. To correct this, they execute Manacher's algorithm [23, Algorithm 1] on $\boldsymbol{w}$ to compute its palindrome array MP $\boldsymbol{x}$. Then they output $\boldsymbol{w}$ if and only if MP $\boldsymbol{x}=$ MP. We on the other hand use a direct approach - our algorithm outputs a regular string if and only if the input MP array is regular. Furthermore, if MP is not regular, their algorithm simply exits, while construct on the other hand yields an indeterminate string.

Consider the example shown in Figure 6. For the given MP array, construct produces the string $\boldsymbol{x}^{*}$. Observe that not all the centres 4-11 satisfy Manacher's condition. Furthermore, if a centre $c$ and a range $k$ fail Manacher's condition, it is not necessarily the case that the centre $c+k$ also fails it. For example, for 


$\begin{array}{ccccccccccccc}1 & 2 & 3 & 4 & 5 & 6 & 7 & 8 & 9 & 10 & 11 & 12 & 13 \\ M P=0 & 1 & 0 & 3 & 2 & 3 & 6 & 3 & 4 & 1 & 2 & 1 & 0 \\ \boldsymbol{x}^{*}=\# & \{1,5\} & \# & \{2,3,4,6\} & \# & \{1,3,7,8,9\} & \# & \{4,7,10\} & \# & \{6,8,10,11\} & \# & \{5,9,11\} & \# \\ \text { FS }=\emptyset & \emptyset & \emptyset & \{2\} & \emptyset & \emptyset & \emptyset & \{2\} & \emptyset & \{2\} & \emptyset & \{4,8\} & \emptyset\end{array}$

Figure 6: Given an MP array that is not regular, procedure construct produces the indeterminate string $\boldsymbol{x}^{*}$.

centre $c=8$, and range $k=3$, Manacher's condition fails; however, the centre $c+k=11$ satisfies it.

As can be observed in the example shown in Figure 6 the number of centres that fail Manacher's condition is of order $n$, where $n$ is the length of the string. Therefore, we are forced to evaluate $\mathcal{O}(n)$ centres in this example. Hence we state the following:

Conjecture 1. To construct an indeterminate string over an MP array of length $m=2 n+1$ that is not regular requires at least $\Omega\left(n^{2}\right)$ time.

\section{Conclusion and Open Problems}

In this paper we have characterized regular strings as those whose triples are all transitive; the strings for which this is not true are called indeterminate. A new approach based on the transitivity of matching among the regular letters of a string enables us to precisely define strings and eliminate false positive cases seen in the traditional definition of indeterminate strings. Based on this new definition, we present a linear time algorithm REGULAR that checks the regularity of the given string $\boldsymbol{x}$ by checking the regularity of its corresponding reduced string $\boldsymbol{x}_{\boldsymbol{R}}$, and as a byproduct yields a lex-least regular string that is isomorphic to $\boldsymbol{x}$ whenever $\boldsymbol{x}$ is regular.

To check the regularity of $\boldsymbol{x}_{\boldsymbol{R}}$, we present two algorithms regular ${ }_{\text {min }}$ and regular $_{\text {min_matrix }}$, which illustrate an interesting connection between the matching relation defined on the letters of $\boldsymbol{x}$ and the computation of the relation's transitive closure. Many algorithms exist to compute the transitive closure of a relation in $\mathcal{O}\left(n^{3}\right)$ time. However, it is a long standing open problem whether 
this complexity can be reduced. We show that for important special cases, the transitive closure can be computed in $\mathcal{O}\left(n^{2}\right)$ time using the regular min_matrix function.

Furthermore, we consider the reverse engineering problem of constructing a string (regular or indeterminate) from the given palindrome array. We present the algorithm construct to output a lex-least regular string, if it exists, in $\mathcal{O}(n)$ time; otherwise the algorithm returns an indeterminate string in $\mathcal{O}\left(n^{2}\right)$ time. An immediate question arises whether an algorithm faster than construct exists to output an indeterminate string. It would also be interesting to develop an algorithm that yields an indeterminate string over a minimum alphabet, corresponding to an MP array that is not regular. Furthermore, although the running time complexity of REGULAR is $\mathcal{O}(n)$, the constant hidden in it could in extreme cases be rather large - is it possible to reduce the size of this constant?

More generally, a new definition of regularity in strings may affect the relationship between strings and the data structures mentioned in the Introduction that facilitate their use: border array, cover array, prefix array, and suffix array. A string containing indeterminate letters may now actually be regular, provided all its triples are transitive: can these important arrays be computed accordingly in linear time?

Conversely, what effect does this new definition have on the "reverse engineering" problem related to these arrays? We have presented in Section 4 an algorithm that reverse engineers the palindrome array to a regular string, whenever possible. There exist algorithms to reverse engineer the other array noted above - can they be modified/extended to yield equivalent results? Can the time requirements of these algorithms be reduced to near-linear, except in pathological cases?

\section{Acknowledgments}

We thank the reviewers for carefully reading the manuscript and providing insightful comments which led to substantial improvements in the manuscript. 
Funding:. Louza was supported by Grant \#2017/09105-0 from the São Paulo Research Foundation (FAPESP). Mhaskar and Smyth were supported by Grant 36797 from the Natural Sciences \& Engineering Research Council of Canada (NSERC).

\section{References}

[1] M. Fischer, M. Paterson, String matching and other products, in: R. Karp (Ed.), Complexity of Computation,, American Mathematical Society, 1974, pp. $113-125$.

[2] K. Abrahamson, Generalized string matching, SIAM Journal of Computing 16 (6) (1987) 1039-1051.

[3] F. Blanchet-Sadri, Algorithmic Combinatorics on Partial Words, Chapman \& Hall CRC, 2008.

[4] W. F. Smyth, S. Wang, A new approach to the periodicity lemma on strings with holes, Theoret. Comput. Sci. 410 (43) (2009) 4295 - 4302.

[5] C. S. Iliopoulos, L. Mouchard, M. S. Rahman, A new approach to pattern matching in degenerate DNA/RNA sequences and distributed pattern matching, Math. in Computer Science (2008) 557-569.

[6] C. Iliopoulos, M. S. Rahman, M. Voráček, L. Vagner, Finite automata based algorithms on subsequences and supersequences of degenerare strings, J. Discrete Algorithms 6 (2) (2010) 117-130.

[7] C. Iliopoulos, R. Kundu, S. P. Pissis, Efficient pattern-matching in elasticdegenerate strings, Proc. Language \& Automata Theory \& Applications (LATA 2017), LNCS 10168 (2017) 131-142.

[8] J. Holub, W. F. Smyth, Algorithms on indeterminate strings, Proc. 14th Australasian Workshop on Combinatorial Algs. (AWOCA) (2003) 36-45. 
[9] J. Holub, W. F. Smyth, S. Wang, Fast pattern-matching on indeterminate strings, Proc. 16th Australasian Workshop on Combinatorial Algorithms (2005) 415-428.

[10] J. Holub, W. F. Smyth, S. Wang, Hybrid pattern-matching algorithms on indeterminate strings, in: J. W. Daykin, M. Mohamed, K. Steinhofel (Eds.), London Algorithmics and Stringology, King's College Texts in Algorithmics, 2006, pp. 115-133.

[11] J. Holub, W. F. Smyth, S. Wang, Fast pattern-matching on indeterminate strings, J. Discrete Algorithms 6 (1) (2008) 37-50.

[12] W. F. Smyth, S. Wang, An adaptive hybrid pattern-matching algorithm on indeterminate strings, Internat. J. Foundations of Computer Science 20 (6) (2009) 985-1004.

[13] S. Nazeen, S. M. Rahman, R. Reaz, Indeterminate string inference algorithms, J. Discrete Algorithms 10 (2012) 23-34.

[14] M. F. Bari, M. S. Rahman, R. Shahriyar, Finding all covers of an indeterminate string in $o(n)$ time on average, in: J. Holub, J. Ždárek (Eds.), Proceedings of the Prague Stringology Conference 2009, 2009, pp. 263-271.

[15] A. Alatabbi, M. S. Rahman, W. F. Smyth, Inferring an indeterminate string from a prefix graph, J. Discrete Algorithms 32 (2015) 6-13.

[16] M. Christodoulakis, P. J. Ryan, W. F. Smyth, S. Wang, Indeterminate strings, prefix arrays and undirected graphs, Theoretical Comput. Sci. 600 (4) (2015) 34-48.

[17] H. Bannai, S. Inenaga, A. Shinohara, M. Takeda, Inferring strings from graphs and arrays., in: B. Rovan, P. Vojt's (Eds.), Symp. on Mathematical Foundations of Computer Science, Vol. 2747 of Lecture Notes in Computer Science, Springer, 2003, pp. 208-217. 
[18] J. Helling, P. J. Ryan, W. F. Smyth, M. Soltys, Constructing an indeterminate string from its associated graph, Theoret. Comput. Sci. 710 (2018) $88-96$.

[19] F. Franek, W. Lu, P. J. Ryan, W. F. Smyth, Y. Sun, L. Yang, Verifying a border array in linear time (preliminary version), Proc. 10th Australasian Workshop on Combinatorial Algs. (AWOCA) School of Computing, Curtin University of Technology (1999) 26-33.

[20] F. Franek, S. Gao, W. Lu, P. J. Ryan, W. F. Smyth, Y. Sun, L. Yang, Verifying a border array in linear time, J. Combinatorial Maths. and Combinatorial Comput. 42 (2002) 223-236.

[21] J. Clément, M. Crochemore, G. Rindone, Reverse engineering prefix tables, in: Proc. 26th Symp. on Theoretical Aspects of Computer Science, 2009, pp. $289-300$.

[22] J. W. Daykin, F. Franek, J. Holub, A. S. M. S. Islam, W. F. Smyth, Reconstructing a string from its Lyndon arrays, Theoret. Comput. Sci. 710 (2018) 44-51.

[23] T. I, S. Inenaga, H. Bannai, M. Takeda, Counting and verifying maximal palindromes, Proc. 17th Symposium on String Proocessing \& Information Retrieval (SPIRE), LNCS 6393 (2010) 135-146.

[24] P. Procházka, J. Holub, On-line searching in IUPAC nucleotide sequences, in: E. D. Maria, A. L. N. Fred, H. Gamboa (Eds.), Proceedings of the 12th International Joint Conference on Biomedical Engineering Systems and Technologies (BIOSTEC 2019) - Volume 3: BIOINFORMATICS, Prague, Czech Republic, February 22-24, 2019, SciTePress, 2019, pp. 66-77.

[25] L. Huang, V. Popic, S. Batzoglou, Short read alignment with populations of genomes, Bioinformatics 29 (13) (2013) i361-i370.

[26] F. Gray, Pulse code communication. 
[27] T. H. Cormen, C. E. Leiserson, R. L. Rivest, C. Stein, Introduction to Algorithms, 2nd Edition, MIT Press, Cambridge, MA, 2001.

[28] R. W. Floyd, Algorithm 97: Shortest path, Communications of the ACM 5 (6) (1962) 345.

[29] G. Manacher, A new linear-time on-line algorithm for finding the smallest initial palindrome of a string, J. Assoc. Comput. Mach. 22 (1975) 346-351. 